We have proved this theorem, it is true, only when $k>0$. If $k=0$ it is, however, merely an obvious consequence of Theorem I.

We come now at last to our most important result, though one which is, at bottom, less far reaching than Theorem II, namely

THEOREM III. If $\epsilon$ is an arbitrarily given positive constant, a continuous, real function $g(x)$ exists such that $0<g(x)<\epsilon$ and such that the system (5) is incompatible.

The proof consists simply in noticing that if we add to the function $g(x)$ determined in Theorem II a sufficiently small function everywhere positive (not zero), the system (5) will, by Theorem I, remain incompatible.*

This theorem is useful in making connection, by the method originally given in special cases by Hilbert, between the system (1) and an integral equation of the second kind.

HARVARD UNIVERSITY, Cambridge, Mass.

\title{
THE SMALLEST CHARACTERISTIC NUMBERS IN A CERTAIN EXCEPTIONAL CASE.
}

\author{
BY PROFESSOR MAXIME BÔCHER
}

(Read before the American Mathematical Society, September 8, 1914.)

THE characteristic numbers of the system

$$
\begin{aligned}
\frac{d}{d x}\left(k u^{\prime}\right)+(\lambda g-l) u & =0, \quad(k>0, l \geqq 0), \\
\alpha u^{\prime}(a)-\alpha^{\prime} u(a) & =0, \quad\left(\alpha \alpha^{\prime} \geqq 0,|\alpha|+\left|\alpha^{\prime}\right|>0\right), \\
\beta u^{\prime}(b)+\beta^{\prime} u(b) & =0, \quad\left(\beta \beta^{\prime} \geqq 0,|\beta|+\left|\beta^{\prime}\right|>0\right)
\end{aligned}
$$

are those values of $\lambda$ for which (1) has a solution not identically zero which satisfies (2) and (3). We assume that $k, g, l$ are continuous real functions of $x$ in the interval $a \leqq x \leqq b$,

* A similar method enables us to deduce from Theorem II a great variety of other results, for instance:

If $\epsilon$ is an arbitrarily given positive constant, and $x_{1}, \cdots, x_{p}$ are arbitrarily given points in $a b$, there exists a continuous, real function $g(x)$ which vanishes and changes sign at each of the points $x_{i}$ but vanishes nowhere else in $a b$, which satisfies the condition $|g(x)|<\epsilon$, and for which (5) is incompatible. 
and that $\alpha, \alpha^{\prime}, \beta, \beta^{\prime}$ are real constants. It is with the real characteristic values we shall be concerned; indeed it is easily proved that there are no others.

If $g \geqq 0$, but not identically zero, we have obviously a special case of the system considered by Sturm in his first memoir of 1836, and indeed one which Sturm, and later Liouville, considered explicitly. If $g$ changes sign, we have a case which has been supposed to be essentially different from Sturm's, and has been several times treated on this basis by more or less independent methods. I believe I was the first to point out* that if we divide (1) by $|\lambda|$ the system becomes merely a special case of the one originally treated by Sturm, whose results tell us that the characteristic numbers consist of two infinite systems, $\lambda_{0}{ }^{+}, \lambda_{1}{ }^{+}, \cdots$ and $\lambda_{0}{ }^{-}, \lambda_{1}{ }^{-}, \cdots$, the first set positive, the second negative (we suppose these sets to be both arranged according to increasing absolute values), such that the characteristic function corresponding to any one of them has in the interval $a<x<b$ a number of zeros exactly equal to the subscript of the $\lambda$ in question.

There is, however, one exceptional case in which the result just stated becomes accurate only after a slight modification, namely that in which

$$
l \equiv 0, \quad \alpha^{\prime}=\beta^{\prime}=0 .
$$

In this case, and in this case only, $\lambda=0$ is a characteristic number, a corresponding characteristic function being $u=1$. In this case also Sturm's results assert the existence of the characteristic numbers $\lambda_{i}^{+}$and $\lambda_{i}^{-}$, when $i \geqq 1$, and the fact that the corresponding characteristic functions vanish just $i$ times. It is not, however, immediately obvious whether the numbers $\lambda_{0}{ }^{+}$and $\lambda_{0}{ }^{-}$still exist. As a matter of fact at least one, and sometimes both of them, will have disappeared, or, if we prefer, will reduce to the value $\lambda=0$. The facts in this special case were established by Picone $\nmid$ by a different method. I wish here to show how very readily they follow by means of Sturm's results.

\footnotetext{
* In a lecture delivered at the 5th International Congress of Mathematicians at Cambridge, England, 1912. See Proceedings, vol. 1, pp. 173, 176. The literature there cited should have included a reference to Hilb, Jahresbericht der deutschen Mathematiker-Vereinigung, vol. 16 (1907), p. 279, where a sketch for a treatment of a special case in which $g$ changes sign is given, viz. $l \equiv 0, \alpha=\beta=0$.

$\dagger$ Annali della R. Scuola Normale di Pisa, vol. 11 (1909), p. 39.
} 
Let $v(x, \lambda)$ be the solution of (1) for which $v(a, \lambda)=1$, $v^{\prime}(a, \lambda)=0$. According to Sturm's second theorem of comparison, * as $\lambda$ increases from the value 0 , the function

$$
\frac{k(b) v^{\prime}(b, \lambda)}{\lambda v(b, \lambda)}
$$

decreases until it becomes negatively infinite. This occurs when $\lambda$ reaches the value, $\mu_{1}$, for which $v\left(b, \mu_{1}\right)=0$. The characteristic number $\lambda_{0}{ }^{+}$, if it exists, must evidently lie in the interval $0<\lambda<\mu_{1}$, since a characteristic function corresponding to a characteristic number greater than $\mu_{1}$ would necessarily have at least one zero in $a b$.

Now (4) approaches a definite finite limit as $\lambda$ approaches zero, namely

$$
D=k(b)\left[\frac{\partial}{\partial \lambda} v^{\prime}(b, \lambda)\right]_{\lambda=0} .
$$

Consequently, if $D>0$, the quantity (4) in its decrease starts from positive values and passes, for some value of $\lambda$ less than $\mu_{1}$, through the value zero. This is the characteristic value $\lambda_{0}{ }^{+}$, since for it $v^{\prime}(b, \lambda)=0$. On the other hand, if $D \leqq 0$, (4) does not pass through the value zero, and $\lambda_{0}{ }^{+}$does not exist.

In just the same way, by considering negative values of $\lambda$ and dividing (1) by $|\lambda|$, we infer from Sturm's second theorem of comparison that, if $D<0, \lambda_{0}{ }^{-}$exists, if $D \geqq 0$, it does not.

It remains merely to evaluate $D$ in terms of given quantities. From (1) we have

$$
\frac{\partial}{\partial x}\left[k(x) v^{\prime}(x, \lambda)\right]=-\lambda g(x) v(x, \lambda) .
$$

Integrating, and remembering that $v^{\prime}(a, \lambda)=0$, we get

$$
k(b) v^{\prime}(b, \lambda)=-\lambda \int_{a}^{b} g(x) v(x, \lambda) d x
$$

or, on differentiating with regard to $\lambda$,

$k(b) \frac{\partial}{\partial \lambda} v^{\prime}(b, \lambda)=-\int_{a}^{b} g(x) v(x, \lambda) d x-\lambda \int_{a}^{b} g(x) \frac{\partial}{\partial \lambda} v(x, \lambda) d x$.

If here we let $\lambda=0$, we get the desired formula

$$
D=-\int_{a}^{b} g(x) d x
$$

\footnotetext{
* See, for instance, Transactions, vol. 1 (1900), p. 420 .
} 
All the steps we have here taken are justified since $v(x, \lambda)$ and $v^{\prime}(x, \lambda)$ are both continuous in $(x, \lambda)$ and analytic in $\lambda_{. *}{ }^{*}$

We have thus established Picone's result:

The differential system

$$
\begin{gathered}
\frac{d}{d x}\left(k u^{\prime}\right)+\lambda g u=0, \quad(k>0) \\
u^{\prime}(a)=0, \quad u^{\prime}(b)=0
\end{gathered}
$$

in which g changes sign in ab has, if $\int_{a}^{b} g d x=0$, no characteristic number other than zero for which the characteristic function does not vanish, otherwise it has just one such characteristic number, namely a positive one if $\int_{a}^{b} g d x<0$, a negative one if $\int_{a}^{b} g d x>0$. I note in closing that the case $\int_{a}^{b} g d x=0$ is of interest as giving one of the simplest examples of a characteristic number $(\lambda=0)$ whose order of multiplicity when regarded as a root of the characteristic equation ( 2 in this case) is not equal to its index ( 1 in this case), i. e., the number of linearly independent characteristic functions corresponding to it.

HARVARD UNIVERSITY,

CAMBRidGe, Mass., July $15,1914$.

\section{ON APPROXIMATION BY TRIGONOMETRIC SUMS.}

BY PROFESSOR T. H. GRONWALL.

(Read before the American Mathematical Society, December 31, 1913.)

IN his paper "On approximation by trigonometric sums and polynomials" $\dagger$ Dr. Jackson has shown that, $f(x)$ being a function of period $2 \pi$ and satisfying the Lipschitz condition

$$
\left|f\left(x_{2}\right)-f\left(x_{1}\right)\right| \leqq \lambda\left|x_{2}-x_{1}\right|
$$

* For the fundamental properties of this important class of functions see my paper "On semi-analytic functions of two variables," Annals of Mathematics, 2d ser., vol. 12 (1910), p. 18. I was not aware when I published this article that some of these properties had been already given by Dini, Annali di Matematica, ser. 3, vol. 12 (1906), p. 179.

$\dagger$ Transactions, vol. 13 (1912), pp. 491-515. 